\title{
Newborn Metabolic Screening: Reference Diagnostic Method
} Evaluation

\section{ART ICLE INF O}

\section{Article Type}

Systematic Review

\section{Authors}

Samari S. ${ }^{1} M S C$,

Younesi B. ${ }^{1} B S c$,

Lashgary B. ${ }^{1} B S C$,

Asiabanha Rezaee M.*PhD

\section{How to cite this article}

Samari S, Younesi B, Lashgary B, Asiabanha Rezaee M. Newborn Metabolic Screening: Reference Diagnostic Method Evaluation. Sarem Journal of Reproductive Medicine. 2017;1(4):179-185.

\section{A B S T RA C T}

Introduction Neonatal metabolic disorders are a group of genetic biochemical diseases that cause diseases in the metabolic pathway due to the lack of enzyme function and enzymatic activity. More than a thousand metabolic disorders have been identified, but these diseases have a low prevalence. These diseases should be diagnosed in the pre-emergence period. The clinical symptoms of these diseases are complex and different. Neonatal screening is conducted for the early and rapid diagnosis of a large number of congenital and metabolic genetic diseases with the aim of identifying and managing the affected baby to prevent complications, death, and disability associated with a hereditary metabolic disorder. In this study, the related articles were searched both in Persian and English, using PubMed, Elsevier, and Google scholar databases. Finally, 38 articles were downloaded, translated, and analyzed in depth, and the history of neonatal metabolic disorder, its clinical in children and adults, as well as recent advances in the field of early diagnosis of these diseases were examined.

Conclusion Today, the use of tandem mass spectrometric methods for the analysis of metabolic disorders has increased our ability to diagnose metabolic mediators and to identify and diagnose a large number of metabolic disorders with one sample and a single analytical run. A tandem mass spectrometric method can detect more than 50 metabolic disorders in just 2 to 3 minutes with a droplet of blood clot on paper.

\section{Keywords Neonatal Screening; Metabolic Diseases; Tandem Mass Spectrometry}

\section{CIT A T ION LIN KS}

[1] The incidence of inherited metabolic disorders in the west ... [2] Newborn screening compared to clinical identification of biochemical genetic ... [3] Newborn screening of inherited metabolic disorders by tandem mass spectrometry ... [4] Inherited metabolic diseases: A clinical ... [5] Screening of newborn infants for metabolic disease ... [6] Inborn errors of metabolism as a cause of neurological disease in adults ... [7] Rare inborn errors of metabolism in adults ... [8] Glycogen storage disease diagnosed in ... [9] Inherited disorders of neurotransmitters ... [10] Endocrine manifestations related to ... [11] Therapy insight: Inborn errors of metabolism ... [12] Inherited liver diseases in ... [13] Expanded newborn screening of inherited metabolic ... [14] Principles and practice of mass screening ... [15] Principles and practice of screening ... [16] Expanded newborn screening ... [17] Newborn screening: Toward a uniform screening panel ... [18] Efficacy and outcome of expanded newborn screening for ... [19] Expanded newborn screening: Outcome in screened ... [20] Ethical problems with neonatal ... [21] National academy of clinical biochemistry laboratory ... [22] Neonatal screening for treatable and untreatable disorders: Prospective ... [23] Newborn screening: Experiences in the middle ... [24] Conference report: Second conference of the ... [25] Biochemical screening of 504,049 newborns in Denmark, the Faroe Islands and Greenland ... [26] IImplementation of extended neonatal screening and a metabolic unit in the State of Qatar: Developing and optimizing strategies in cooperation with the Neonatal Screening Center ... [27] Newborn population screening for classic homocystinuria ... [28] The changing face of newborn screening: Diagnosis of inborn ... [29] Four years of expanded newborn screening in Portugal with tandem mass ... [30] Studies on phenylketonuria. 9. Further observations on the effect of phenylalanine restricted diet on patients with ... [31] Newborn screening progress in developing countries--overcoming internal ... [32] Permanent and transient congenital hypothyroidism ... [33] Incidence of Phenylketonuria in Southern ... [34] Incidence of phenylketonuria (PKU) in ... [35] Newborn Screening for inherited metabolic disorders; News ...

\section{Article History}

Received: April 15, 2016

Accepted: October 12, 2016

ePublished: November 15, 2017 
همين رو تاخير در تشخيص و درمان اين بيمارىها منجر به بروز

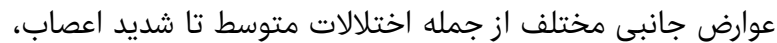

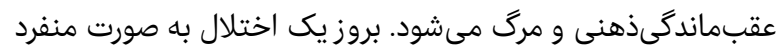

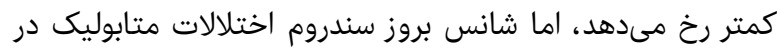

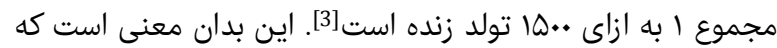

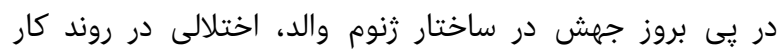

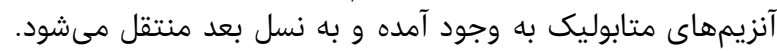

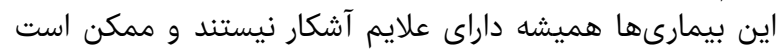

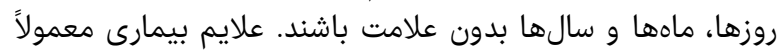

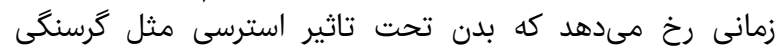

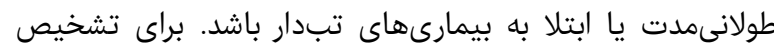

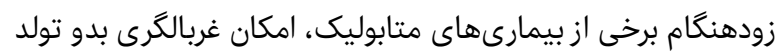

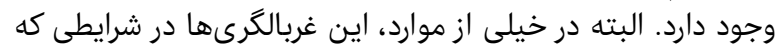

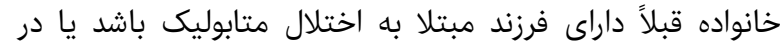

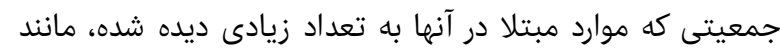
بروز بيمارى تاىساكس در جمعيت يهوديان اشكنازى آنجاد اندام

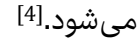
طبقابندى بيمارىهاى متابوليك انواع زيادي إيى دارد. قديمىترين

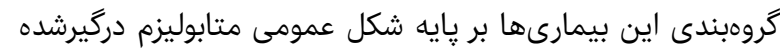

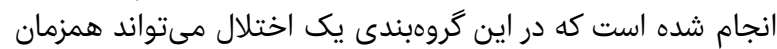

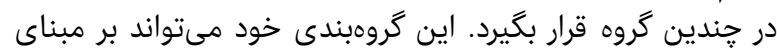

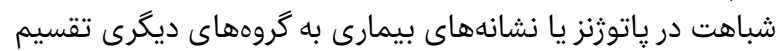

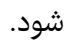

در گروهبندى ديكر اين اختلالات به دو كروه اصلى تقسيم مى مُشوند؛

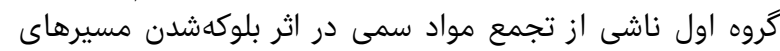

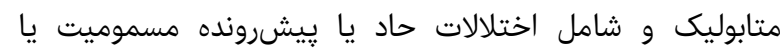

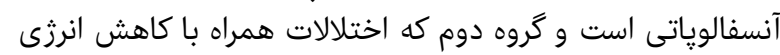

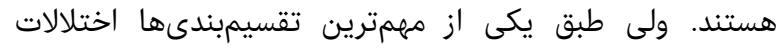

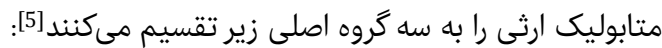

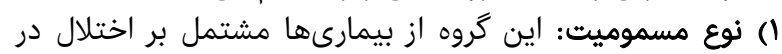

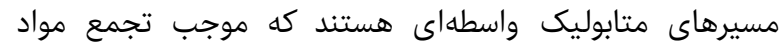

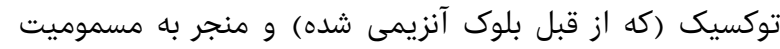

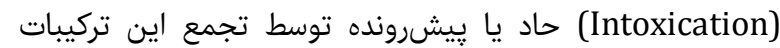

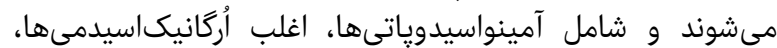

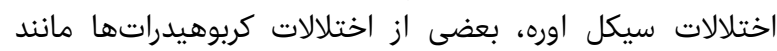

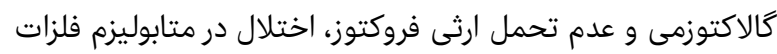

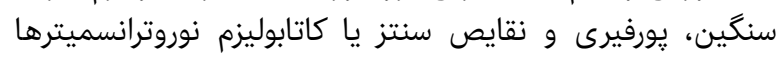

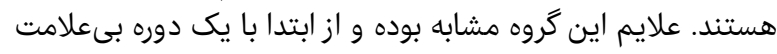

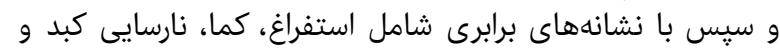

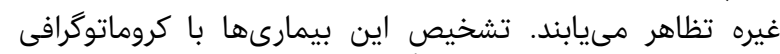

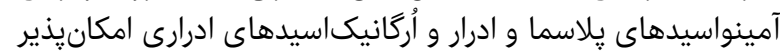

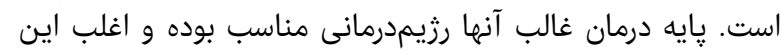

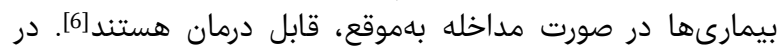

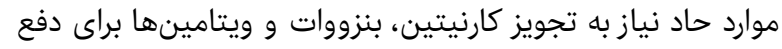
متابوليتهاى توكسيك است.

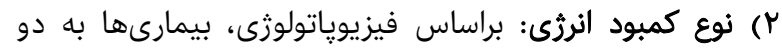

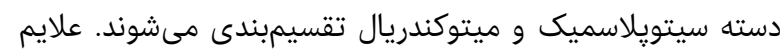

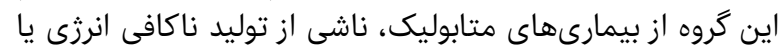

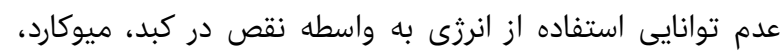

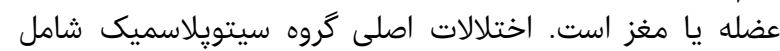

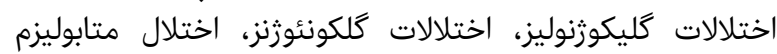
كراتين، اختلال مسير ينتوز و هييرانسولينيسم است و اخلينات علامت

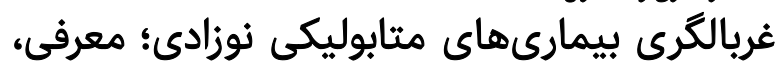
اهميت و روشهاى بررسى آن مئن

سارا ثمرى MSc M

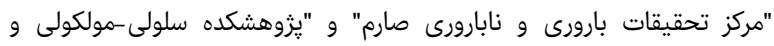

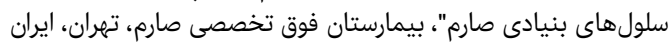

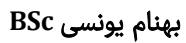

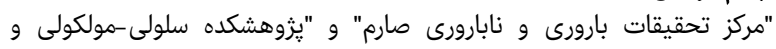

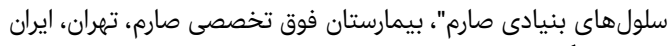

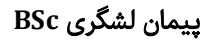

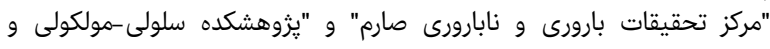

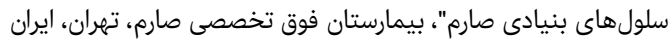

PhD "مجيد آسيابانها رضائي

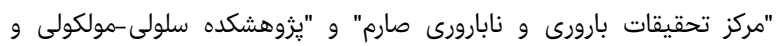
سلولهاى بنيادى صارم"، بيمارستان فوق تخصصى صارئ صارم، تهران، ايران

جكيده

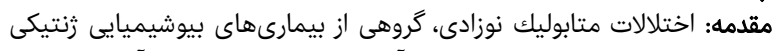

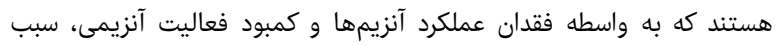

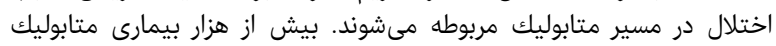

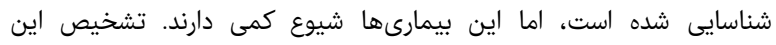

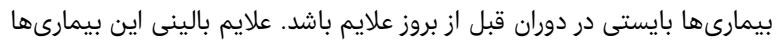

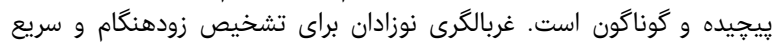

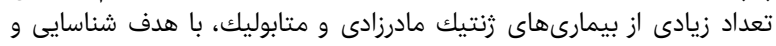

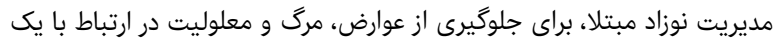

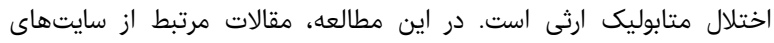

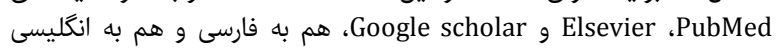

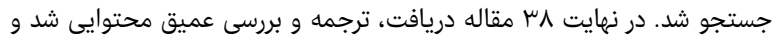

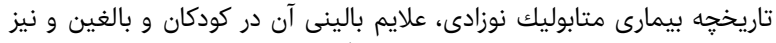

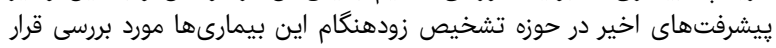

كرفت.

نتيجهگيرى: امروزه استفاده از تكنيك اسيكترومترى جرمى برائ براى آناليز

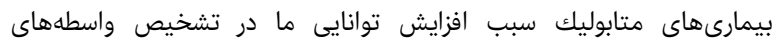

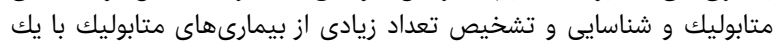

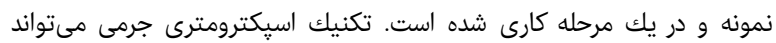

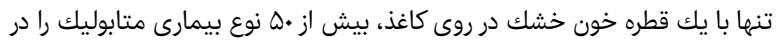
عرض فقط r-r د دقيقه شناسايى نمايد. كليدوارهها: غربالكرى نوزادى، بيقاديمارى متابوليك، اسيكترومترى جرمى

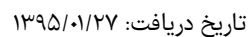

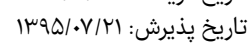
"نويسنده مسئول: mr.asiabanha@gmail.com

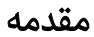

بيمارى متابوليك به اختلالى اطلاق مى شود كه موجب ايجاد

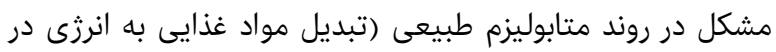

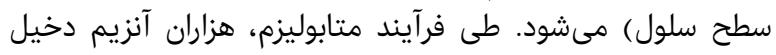

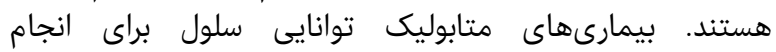

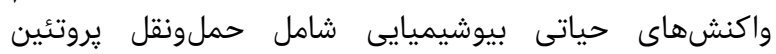

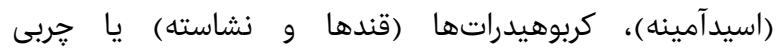

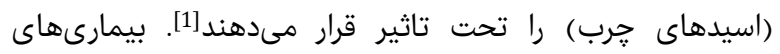

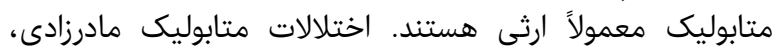

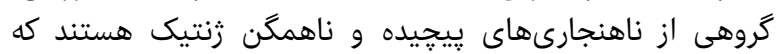

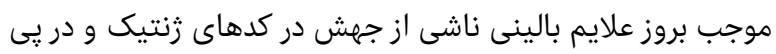

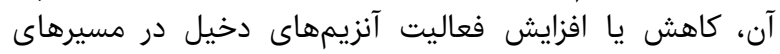

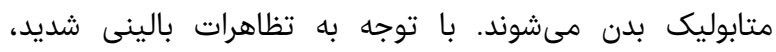

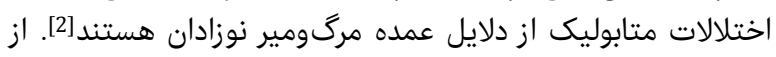




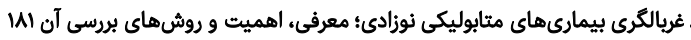

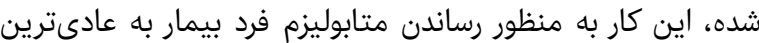

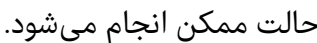
- حذف آن دسته از محصولات سمى مئ كه در اثر اختلال متابوليك، در

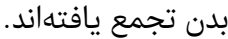
- - رزيم غذايى خاص را را رعايت كنند.

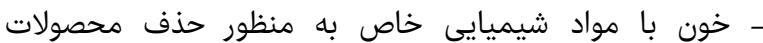
متابوليك توكسيك سمزدايى شود.

جدول () برخى از نشانههاى بيمارىهاى متابوليك إيكائ

\begin{tabular}{|c|c|}
\hline عوارض اختلال مذكور & اختلال ايجادشده در اثر \\
\hline عقبماندگىذهنى & مشكلات رشد \\
\hline 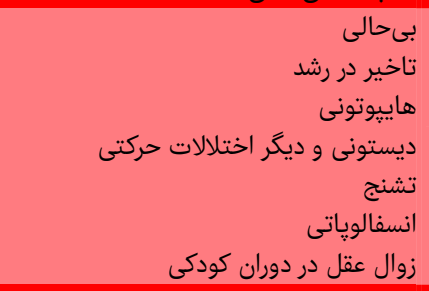 & مشكلات عصبى \\
\hline اوتيسم اخلات رفتارى & مشكلات روانى \\
\hline استهال استفراغ مكرر & مشكلات گوارشى \\
\hline هتوزيدوز & مشكلات بيوشيميايى \\
\hline كاردارديوميوياتى $\begin{array}{r}\text { كاريتمى قلبى } \\
\text { كايتى }\end{array}$ & مشكلات قلبى و عروقى \\
\hline رابدوياتىليز & مشكلات عضلانى-اسكلتى \\
\hline 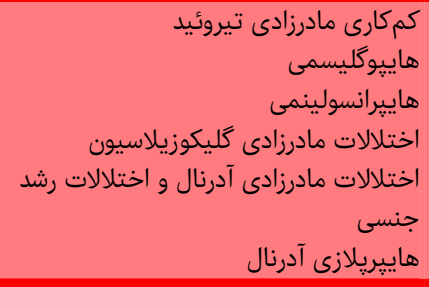 & مشكلات غدد درونريز \\
\hline 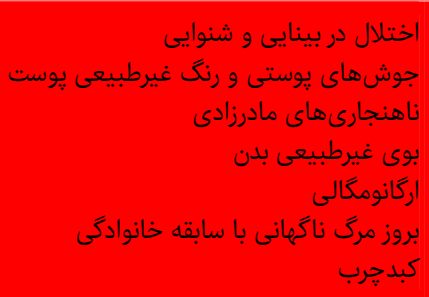 & ساير مشكلات \\
\hline
\end{tabular}

هدف كلى از غربالكرى بدو تولد، تشخيص اختلالات شديد

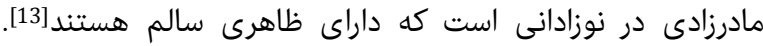

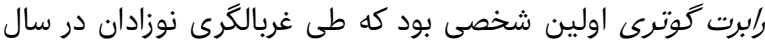

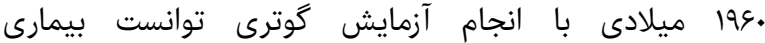

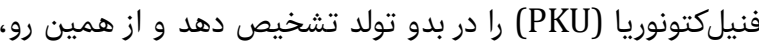

شايع معمول مىتوان از هييوگليسمى نام برد[7]. در گروه

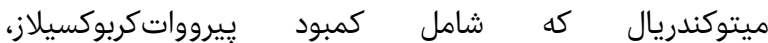

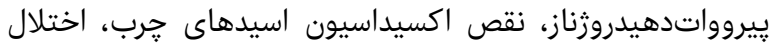

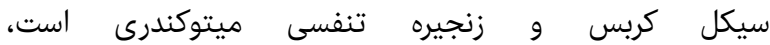

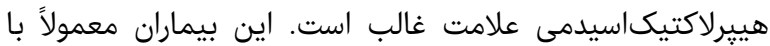

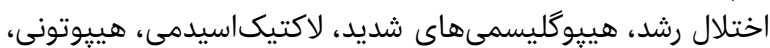

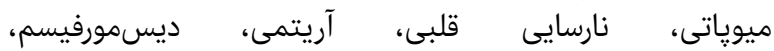

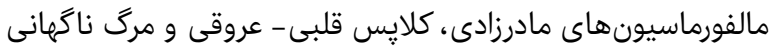

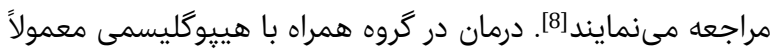

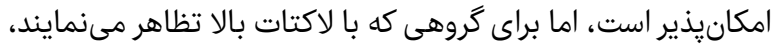

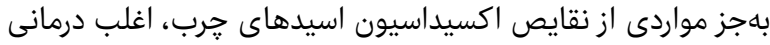

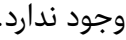

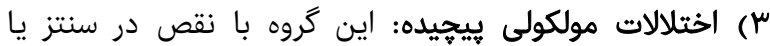

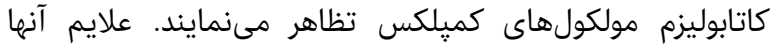

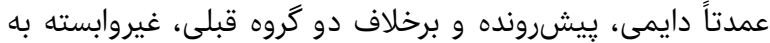

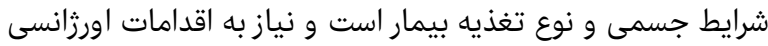

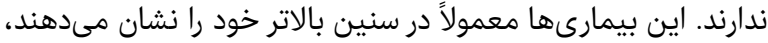

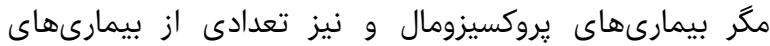

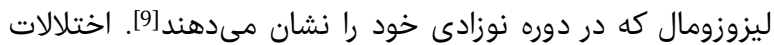

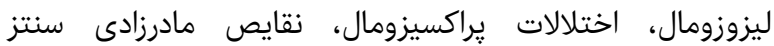

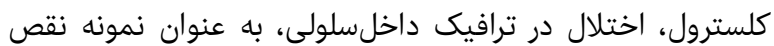

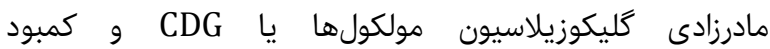

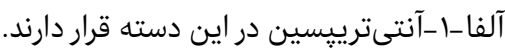

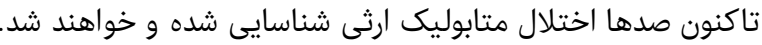

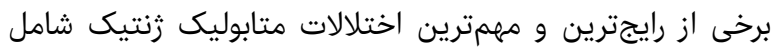

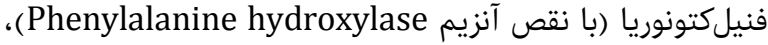
بيمارى شربت افرا (با نقص در نقيم انزيم نقائ (acid dehydrogenase complex

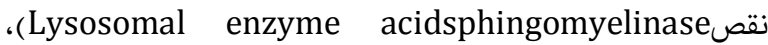
بيمارى تاىساكس (با نقص Beta-N-acetylhexosaminid) و

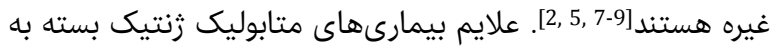

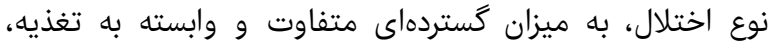

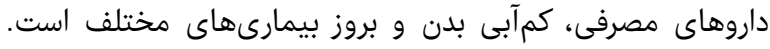

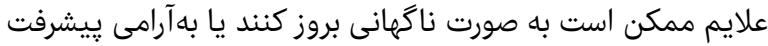

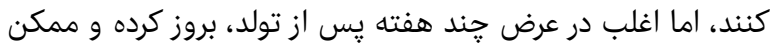

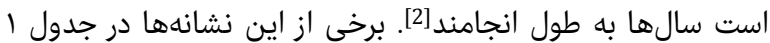

خلاصه شده است[10].

درمانهاى محدودى براى اختلالات متابوليك موجود است. درمان

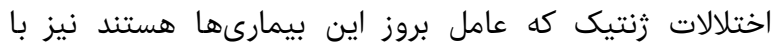

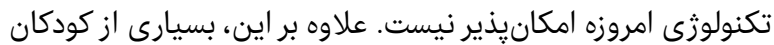

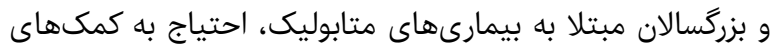

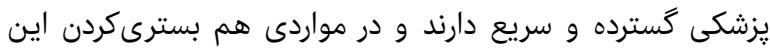

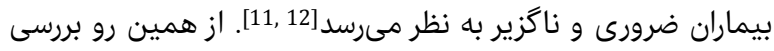

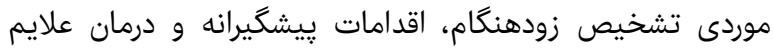

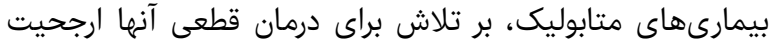

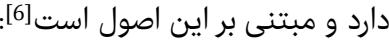
ـ كاهش يا حذف هر مادئ ماده غذائ اهول كه در بدن بيمار بهدرستى

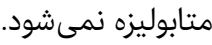
- جايكزينكردن آنزيم يا هر ماده شيميايى ديكرى كه دجار مشكل 
آزمونها براى اضافهشدن به برنامه غربالكرى بدو تولد بيشنهاد

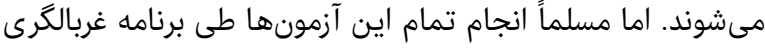

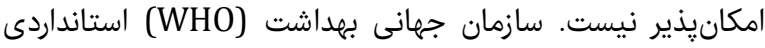

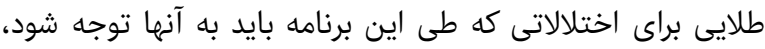

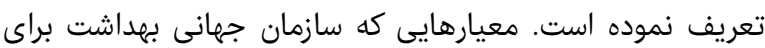

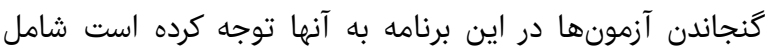

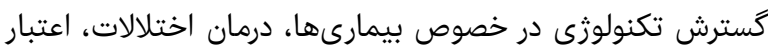

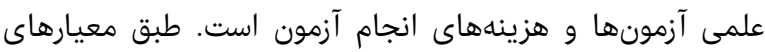

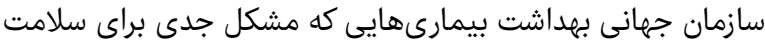

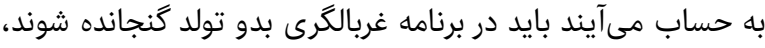

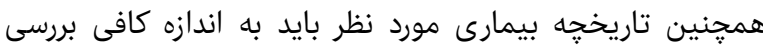

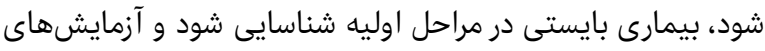

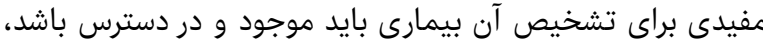

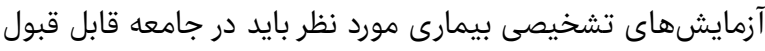

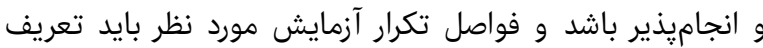

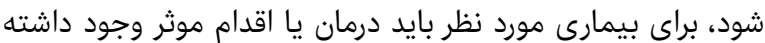

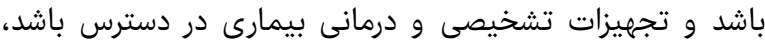

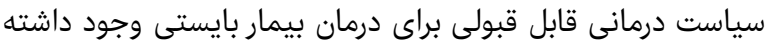
باشد و هزينههاى درمان بايد با منافع دانع حاصل ازئار باز آن همخوانى

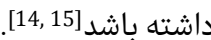

غربالكرى فنيلكتونورى (PKU) براساس برنامهاى انجام مىشد كه براس

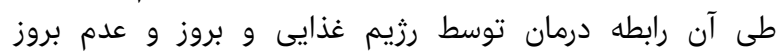

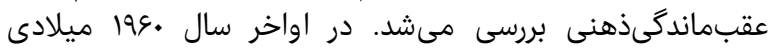

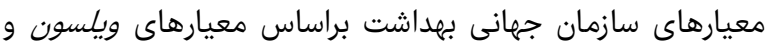

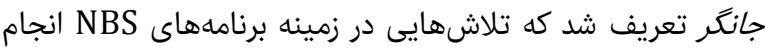

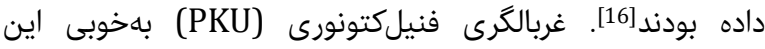

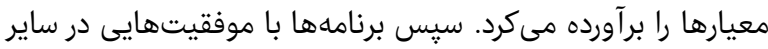

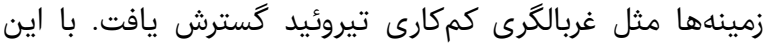

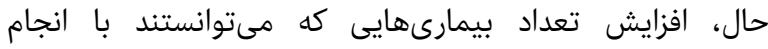

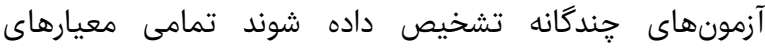

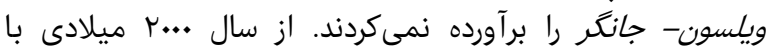

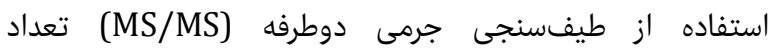

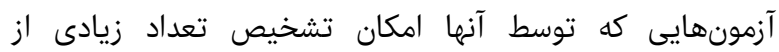

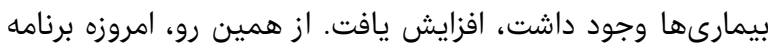

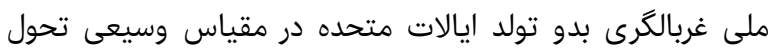

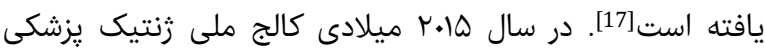

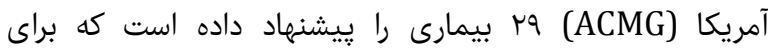

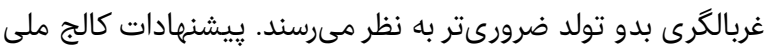

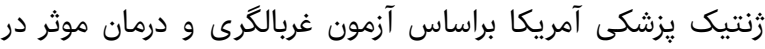

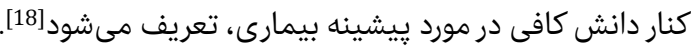

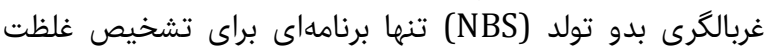

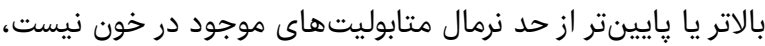

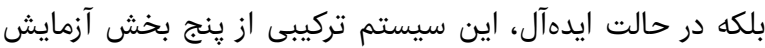

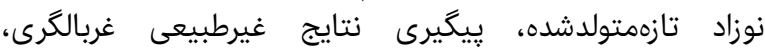

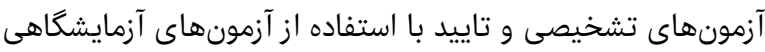

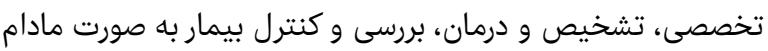

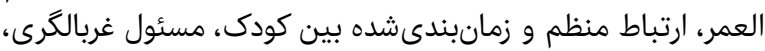

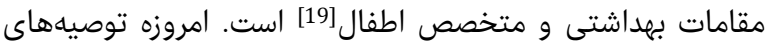

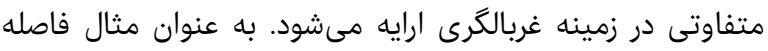

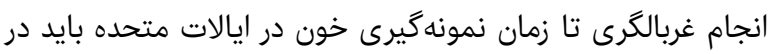

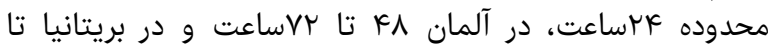

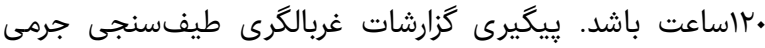

"يدر غربالكرى نوزادان" لقب گرفت[7] (شكل ().

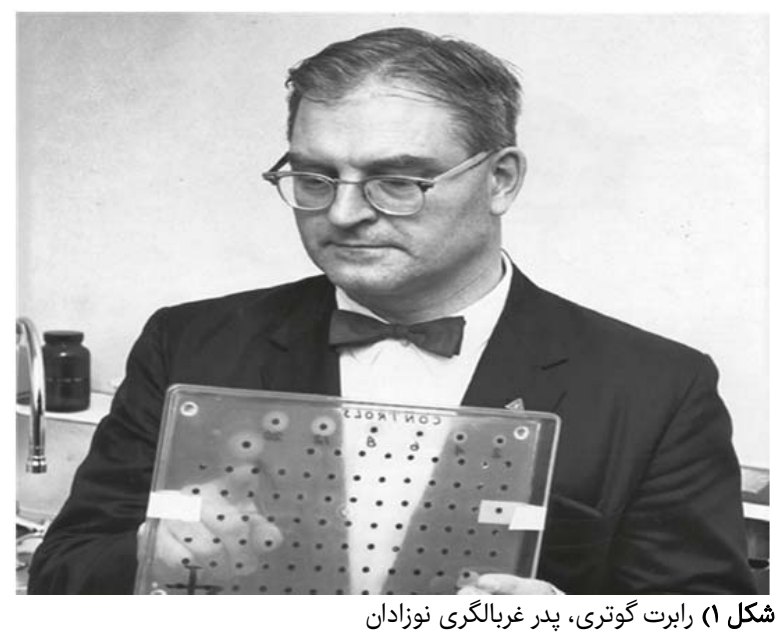

به طور كلى مىتوان كفت بيوماركرها مولكولهايى هستند كه در بردي

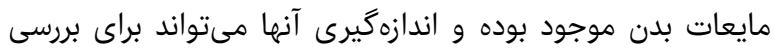

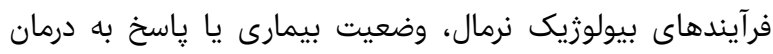

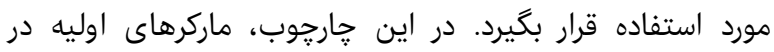

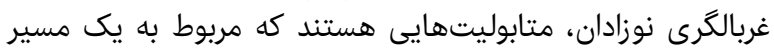

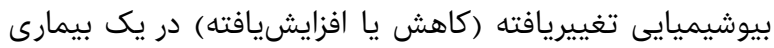

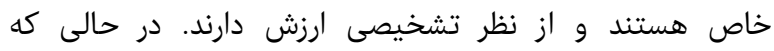

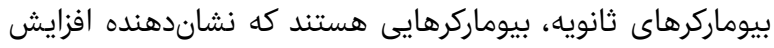

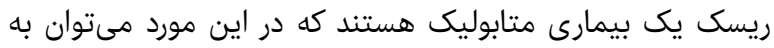

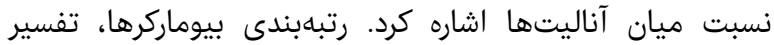

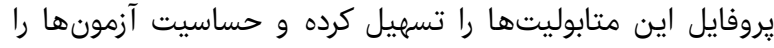

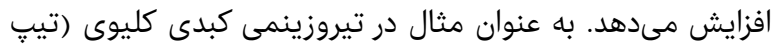

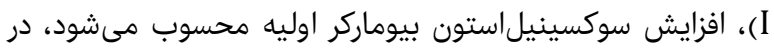

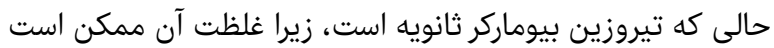

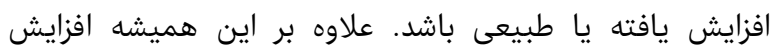

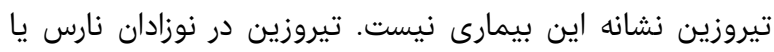

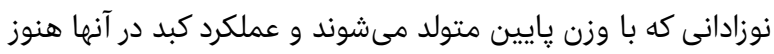

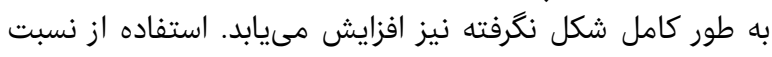

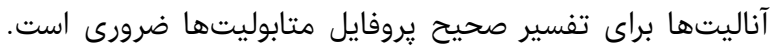

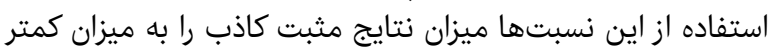

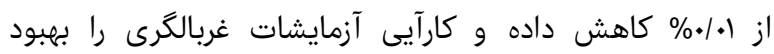

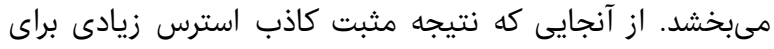

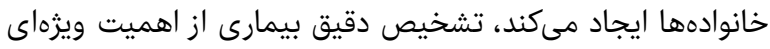

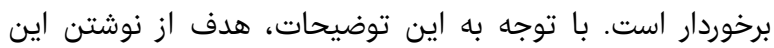

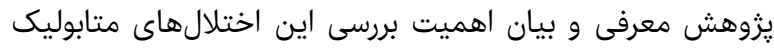

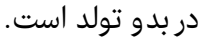

در اين يزوهش از سايتهاى Pubmed، Elsivier و و

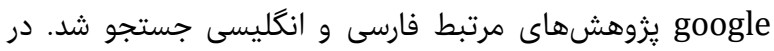

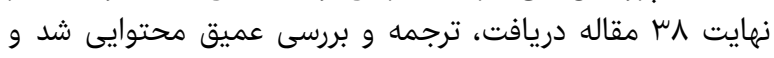

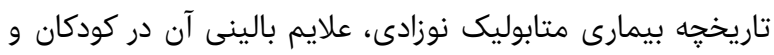

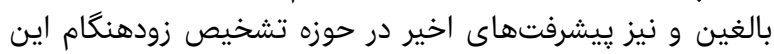

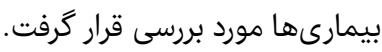

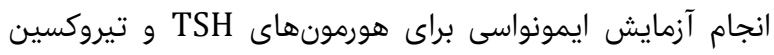

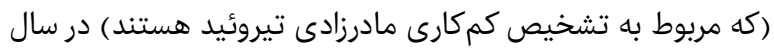
19V.

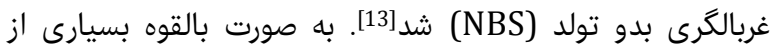




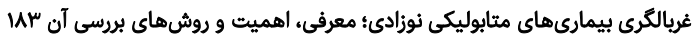

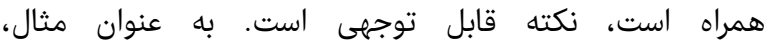

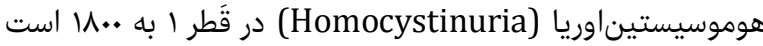

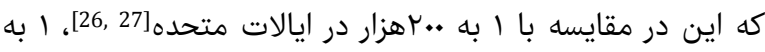

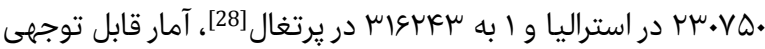

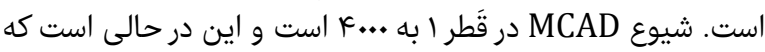

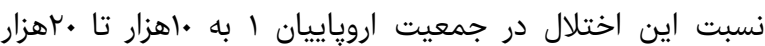

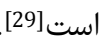

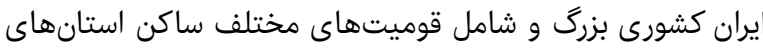

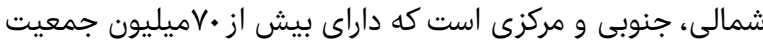

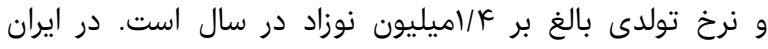

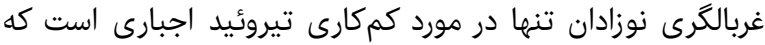

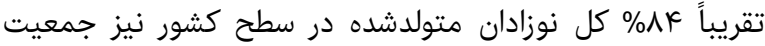

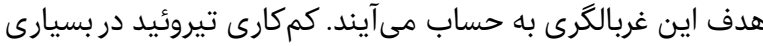

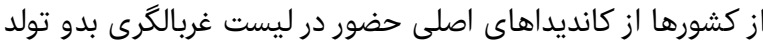

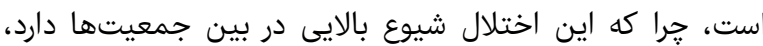

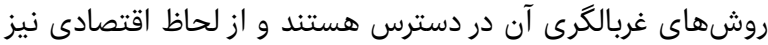

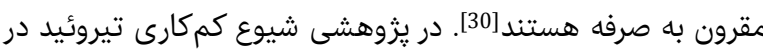

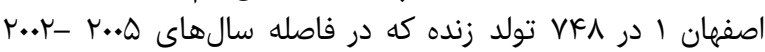

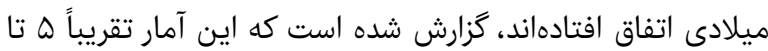

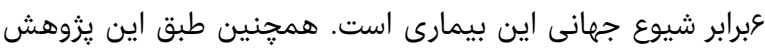

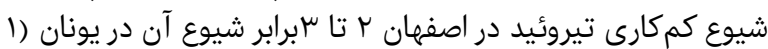

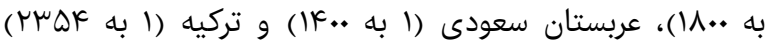

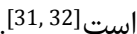

در ايران براى سنجش فنيلكتونورى و كلوكز-4-فسفاتدهيدروزناز،

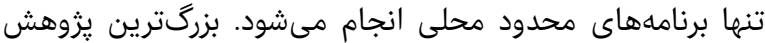

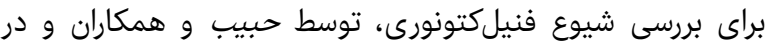

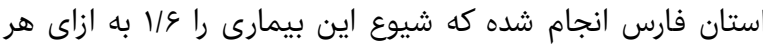

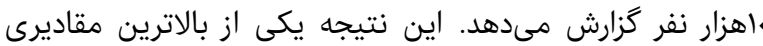

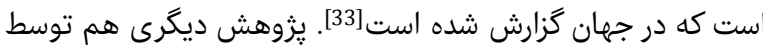

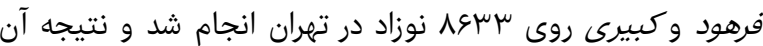

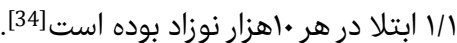

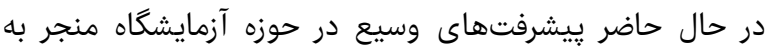

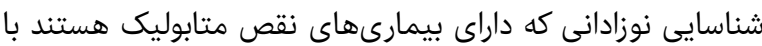

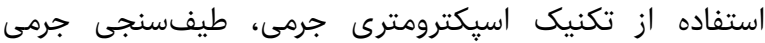

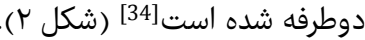

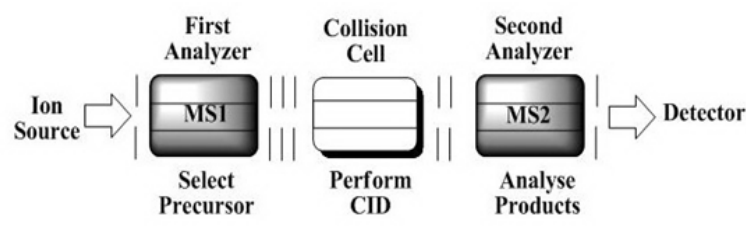

(Tandem Mass شكل Y) اساس و اجزا دستكاه اسيكترومترى جرمى (4) Spectrometry]

در اين تكنيك با يِشرفتهاى حاصلشده، با يك قطره خون

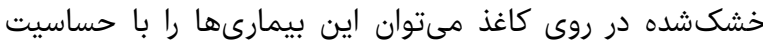

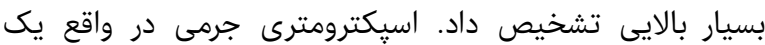

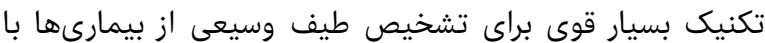

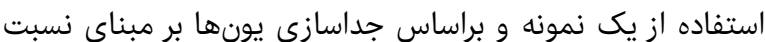

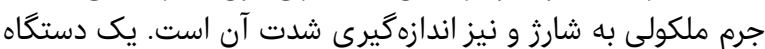

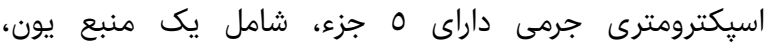

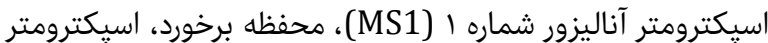

دوطرفه طى يك دوره وساله از كودكان نشان داد كه اين كنترلها

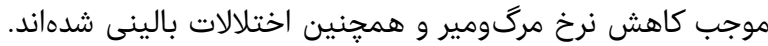

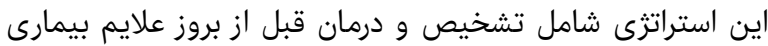

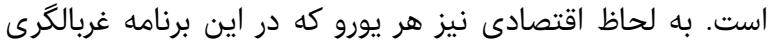

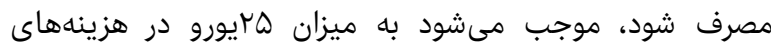

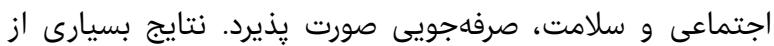

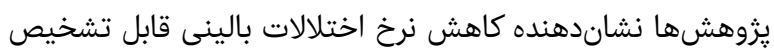

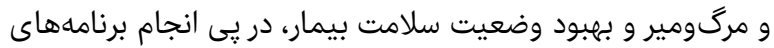

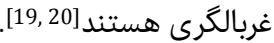

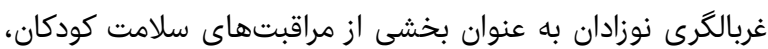

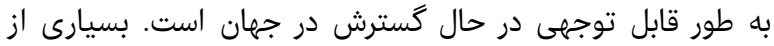

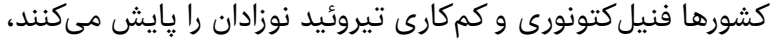

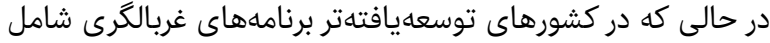

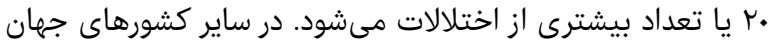

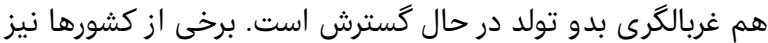
اين برنامه غربالكرى را در سالهاى اخدي اخير آغاز كردهاند.

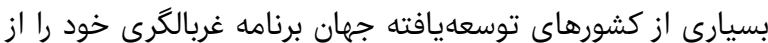

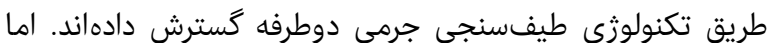

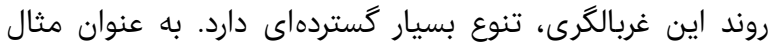

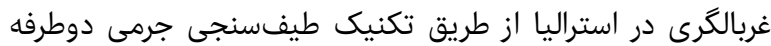

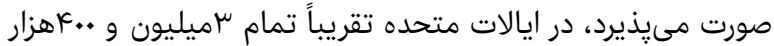

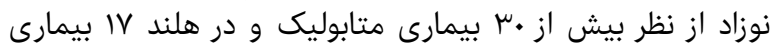

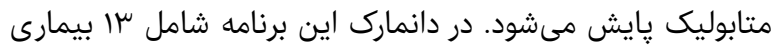

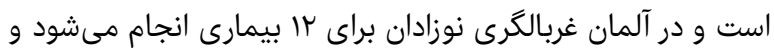

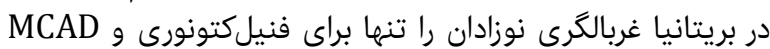

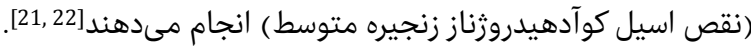

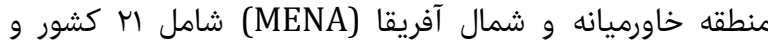

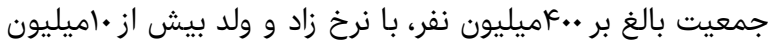

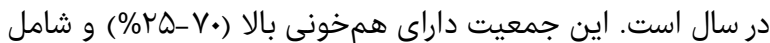

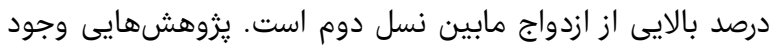

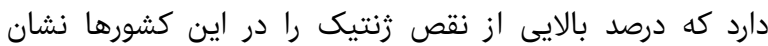

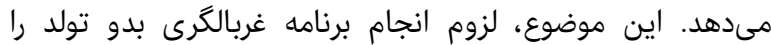

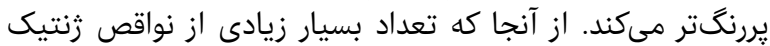

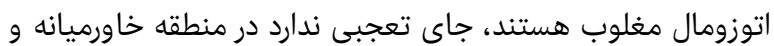

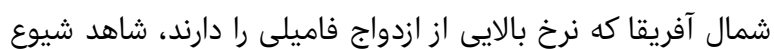

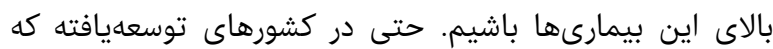

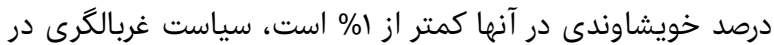

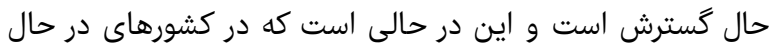

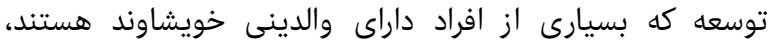

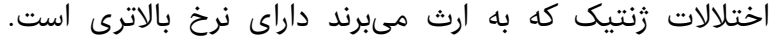

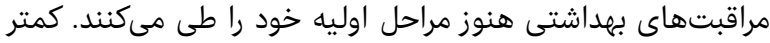

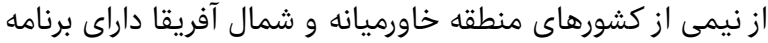

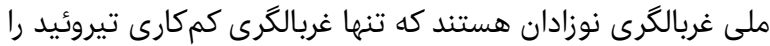

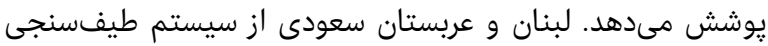

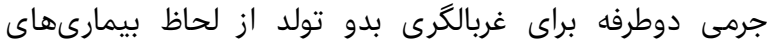

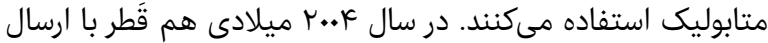

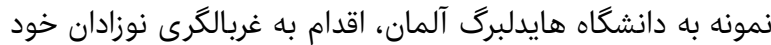

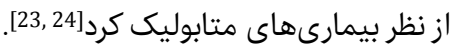

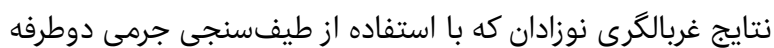

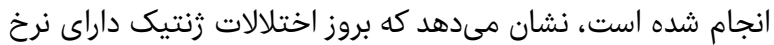

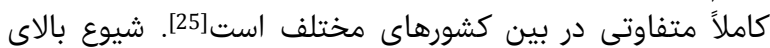
ابتلا به بيمارىهاى متابوليك در قَطر كه با نرخ بالاى همختونى 
تشكر و قدردانى: موردى از سوى نويسندگان ذكر نشده است.

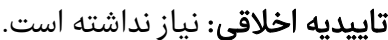

تعارض منافع: موردى وجود نداشته استا نداست.

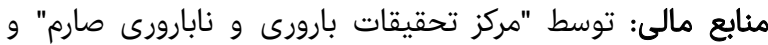

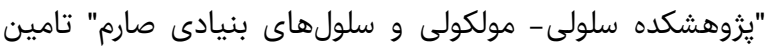

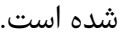

سهم نويسندگان: سارا ثمرى (نويسنده اول)، نكارنده

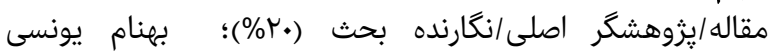

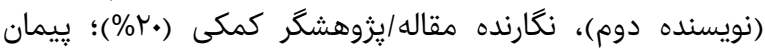

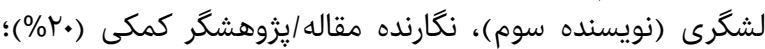

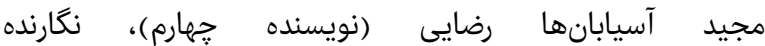

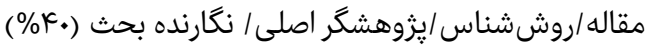

منابع

1- Sanderson S, Green A, Preece MA, Burton H. The incidence of inherited metabolic disorders in the west Midlands, UK. Arch Dis Child. 2006;91:896-9.

2- Waisbren SE, Read CY, Ampola M, Brewster TG, Demmer L. Greenstein $\mathrm{R}$, et al. Newborn screening compared to clinical identification of biochemical genetic disorders. J Inherit Metab Dis. 2002;25(7):599-600.

3- Scaturro G. Sanfilippo C. Piccione M, Piro E, Giuffrè M, Corsello G. Newborn screening of inherited metabolic disorders by tandem mass spectrometry: Past, present and future. Pediatr Med Chir. 2013;35(3):105-9.

4- Hoffmann GF, Zschocke J, Nyhan WL, editors. Inherited metabolic diseases: A clinical approach. Berlin: SpringerVerlag. 2017.

5- Rule JT. Screening of newborn infants for metabolic disease: Committee on fetus and newborn. Pediatrics. 1965;35:499-501.

6- Gray RG, Preece M, Green S, Whitehouse W, Winer J, Green A. Inborn errors of metabolism as a cause of neurological disease in adults: An approach to investigation. J Neurol Neurosurg Psychiatry. 2000;69(1):5-12.

7- Eyskens F. Rare inborn errors of metabolism in adults: The lysosomal storage disorders. Acta Clin Belg. 2009;64(6):534-9.

8- Pears JS, Jung RT, Hopwood D, Waddell ID, Burchell A. Glycogen storage disease diagnosed in adults. Quart J Med. 1992;82(299):207-22.

9- Pearl PL, Capp PK, Novotny EJ, Gibson KM. Inherited disorders of neurotransmitters in children and adults. Clin Biochem. 2005;38(12):1051-8.

10- Vantyghem MC, Dobbelaere D, Mention K, Wemeau JL, Saudubray JM, Douillard C. Endocrine manifestations related to inherited metabolic diseases in adults. Orphanet J Rare Dis. 2012;7:11.

11- Sedel F, Lyon-Caen O, Saudubray JM. Therapy insight: Inborn errors of metabolism in adult neurology--A clinical approach focused on treatable diseases. Nat Clin Pract Neurol. 2007;3(5):279-90.

12- Kumar A, Riely CA. Inherited liver diseases in adults. West J Med. 1995;163(4):382-6.

13- Garg U, Dasouki M. Expanded newborn screening of inherited metabolic disorders by tandem mass spectrometry: clinical and laboratory aspects. Clin Biochem. 2006;39(4):315-32.

14- wilson JM, Jungner YG. Principles and practice of mass screening for disease. Bol Oficina Sanit Panamm. 1968;65(4):281-93. [Spanish]

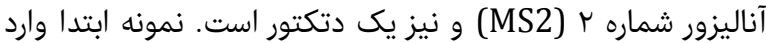

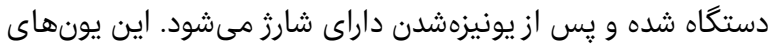
مولكولى به MS1 رسيده و براساس نسبت شارز به جرم جداسازى

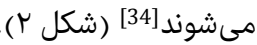
سيس اين يونهاى مولكولى به به محفظه برخورد وارد مئش شوند،

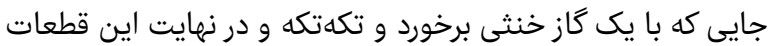

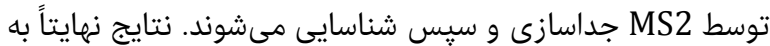

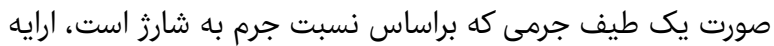

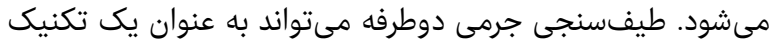

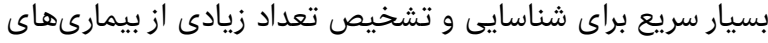

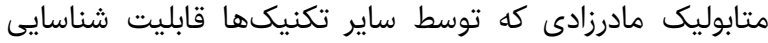

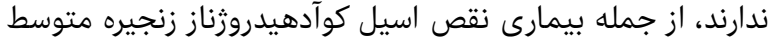

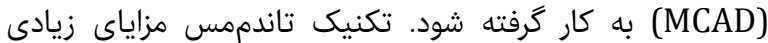

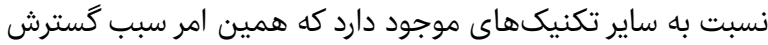

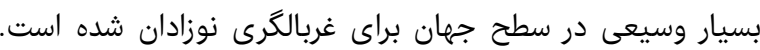

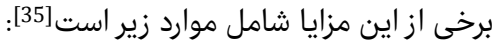
(آ) آناليز مىتواند با مقدايا شامل موارد زير سيت كمى از خون نوزاد يا ساير مايعات

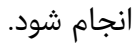

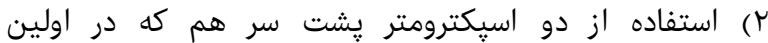

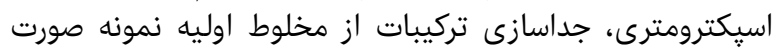

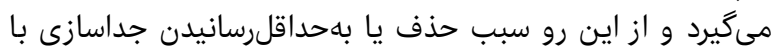

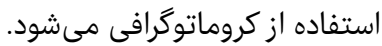

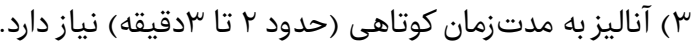

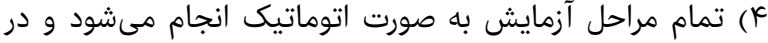

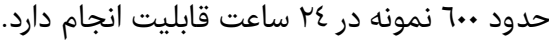

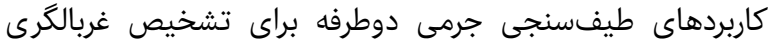

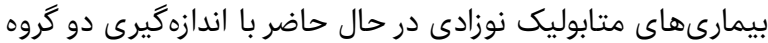

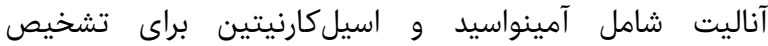

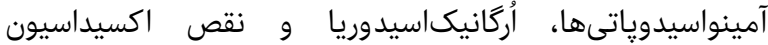

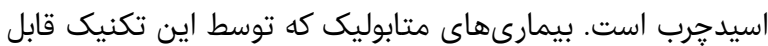

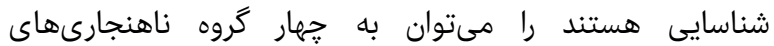

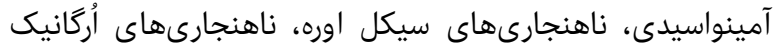

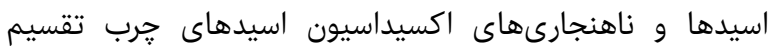

\section{نتيجه گيرى}

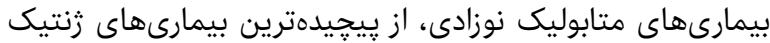

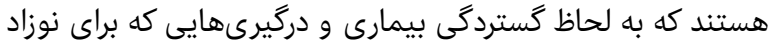

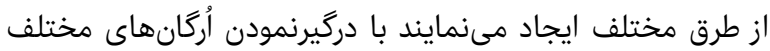

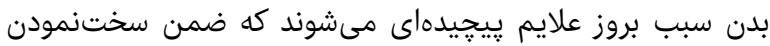

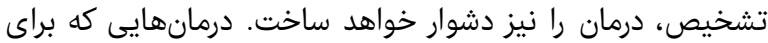

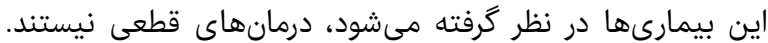

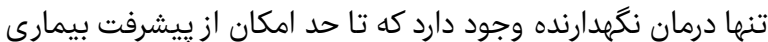

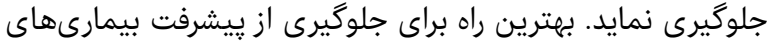

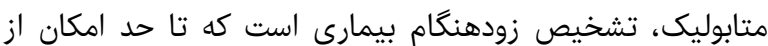

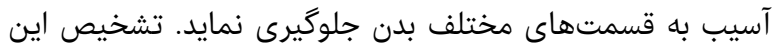

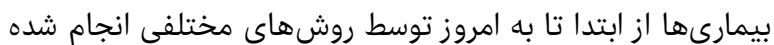

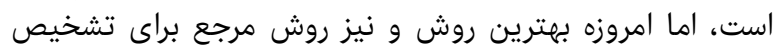

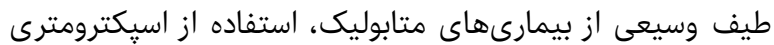

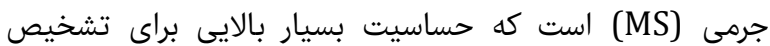
بيمارىهاى متابوليك نوزادى دارد. 
غربالكرى بيمارىهاى متابوليكى نوزادى؛ معرفى، اهميت و روشهاى بررسى آن AM

Christensen M, Duno M, et al. Biochemical screening of 504,049 newborns in Denmark, the Faroe Islands and Greenland--Experienceand development of a routine program for expanded newborns creening. Mol Genet Metab. 2012;107:281-93.

26- Lindner M, Abdoh G, Fang-Hoffmann J, Shabeck N, AlSayrafi M, Al-Janahi M, et al. Implementation of extended neonatal screening and a metabolic unit in the State of Qatar: Developing and optimizing strategies in cooperation with the Neonatal Screening Center in Heidelberg. J Inherit Metab Dis. 2007;30(4):522-9.

27- Gan-Schreier H, Kebbewar M, Fang-Hoffmann J, Wilrich J, AbdohG, Ben-Omran T, et al. Newborn population screening for classic homocystinuria by determination of total homocysteine from Guthrie cards. J Pediatr. 2010;156(3):427-32.

28- Jones PM, Bennett MJ. The changing face of newborn screening: Diagnosis of inborn errors of metabolism by tandem mass spectrometry. Clin Chim Acta. 2002;324(12):121-8.

29- Vilarinho L, Rocha H, Sousa C, Marcao A, Fonseca H, Bogas $M$, et al. Four years of expanded newborn screening in Portugal with tandem mass spectrometry. J Inherit Metab Dis. 2010;33(3 Suppl):S133-8.

30- Armstrong MD, Low NL, Bosma JF. Studies on phenylketonuria. 9. Further observations on the effect of phenylalanine restricted diet on patients with phenylketonuria. Am J Clin Nutr. 1957;5:543-54.

31- Padilla CD, Krotoski D, Therrell BL Jr. Newborn screening progress in developing countries--overcoming internal barriers. Semin Perinatol. 2010;34(2):145-55.

32- Hashemipour M, Hovsepian S, Kelishadi R, Iranpour R, Hadian R, Haghighi S, et al. Permanent and transient congenital hypothyroidism in Isfahan-Iran. J Med Screen. 2009;16(1):11-6.

33- Habib A, Fallahzadeh MH, Kazeroni HR, Ganjkarim $\mathrm{AH}$. Incidence of Phenylketonuria in Southern Iran. Iran J Med Sci. 2010;35(2):137-9.

34- Farhud DD, Kabiri M. Incidence of phenylketonuria (PKU) in Iran. Indian J Pediatr. 1982;49(5):685-8.

35- Pourfarzam M, Zadhoush F. Newborn Screening for inherited metabolic disorders; News and views. J Res Med Sci. 2013;18(9):801-8.
15- Wilson JMG, Junger G. Principles and practice of screening for dsease. Geneva: World Health Organization. 1968.

16- Dhondt JL. Expanded newborn screening: Social and ethical issues. J Inherit Metab Dis. 2010;33(Suppl 2):S211-7.

17- Watson MS, Mann MY, Lloyd-Puryear MA, Rinaldo P, Howell RR. Newborn screening: Toward a uniform screening panel and system--executive summary. Pediatrics. 2006;117(5 Pt 2):S296-307.

18- Lindner M, Gramer G, Haege G, Fang-Hoffmann J, Schwab KO, Tacke U, et al. Efficacy and outcome of expanded newborn screening for metabolic diseasesReport of 10 years from South-West Germany. Orphanet J Rare Dis. 2011;6:44.

19- Wilcken B, Haas M, Joy P, Wiley V, Bowling F, Carpenter $\mathrm{K}$, et al. Expanded newborn screening: Outcome in screened and unscreened patients at age 6 years. Pediatrics. 2009;124(2):e241-8.

20- Orzalesi M, Danhaive O. Ethical problems with neonatal screening. Ann Ist Super Sanita. 2009;45(3):325-30.

21- Dietzen DJ, Rinaldo P, Whitley RJ, Rhead WJ, Hannon WH, Garg UC, et al. National academy of clinical biochemistry laboratory medicine practice guidelines: Follow-up testing for metabolic disease identified by expanded newborn screening using tandem mass spectrometry; executive summary. Clin Chem. 2009;55(9):1615-26.

22- Plass AM, Van El CG, Pieters T, Cornel MC. Neonatal screening for treatable and untreatable disorders: Prospective parents' opinions. Pediatrics. 2010;125(1):e99-106.

23- Saadallah AA, Rashed MS. Newborn screening: Experiences in the middle east and North Africa. J Inherit Metab Dis. 2007;30(4):482-9.

24- Krotoski D, Namaste S, Raouf RK, El Nekhely I, HindiAlexander M, Engelson G, et al. Conference report: Second conference of the Middle East and North Africa newborn screening initiative: Partnerships for sustainable newborn screening infrastructure and research opportunities. Genet Med. 2009;11(9):663-8. 25- Lund AM, Hougaard DM, Simonsen H, Andresen BS, 